\title{
Neutral Triple Vector Boson Production in Randall-Sundrum Model at the LHC
}

\author{
Goutam Das 1, Prakash Mathews 2 \\ Saha Institute of Nuclear Physics, \\ 1/AF Bidhan Nagar, \\ Kolkata 700 064, India
}

\begin{abstract}
In this paper, triple neutral electroweak gauge boson production processes, viz. $\gamma \gamma \gamma, \gamma \gamma Z, \gamma Z Z$ and $Z Z Z$ productions merged to 1-jet have been studied at the leading order in QCD in the context of Randall-Sundrum model at the LHC with center of mass energy $\sqrt{S}=13 \mathrm{TeV}$. Decay of $Z$ bosons into leptonpairs has been considered. We present a selection of kinematical distributions matched to parton shower and show their deviation from the SM results as a result of the RS model. The uncertainties as a result of the factorization and renormalization scales are also presented.
\end{abstract}

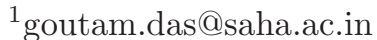

${ }^{2}$ prakash.mathews@saha.ac.in
} 


\section{Introduction}

The Standard Model (SM) of particle physics is now well established as the true description of particles and their interactions at the experimentally accessible energies. The recently discovered $125 \mathrm{GeV}$ scalar at the LHC Run-I [1, 2], behaves like the SM Higgs boson and this fixes the last free parameter of the SM Lagrangian. So far there is no indication of exotic searches of beyond standard model (BSM) physics and the BSM scales have been pushed further. Remarkable agreement between the predicted SM values and the measured cross sections spanning a broad range is a validation of the analytical methods and the Monte Carlo tools developed to match the challenges on the experimental and theoretical sides. None the less the SM is not a complete description of nature on various counts and the issue could only be addressed from beyond [3].

Run-II at the LHC is now on with higher energies and luminosity. Precision measurement of the properties of the newly discovered Higgs boson is a priority and will be matched with precise higher order theoretical predictions to look for any deviations from the SM. Now that the Higgs boson is discovered it is important to look at the massive vector boson scattering (VBS) cross section which is uniterised by the Higgs boson. The VBS gets contribution from the non-abelian couplings of the electroweak gauge boson sector (a) triple gauge boson coupling (TGC), (b) quartic gauge boson coupling (QGC) and in addition, Higgs coupling to the massive gauge boson. The TGC will contribute to the di-boson final state and the Run-I has already placed comparable limits to the anomalous couplings as the LEP experiments. The QGC coupling leads to tri-gauge boson final states, though the full process would involve the TGC, fermion mediated processes and also Higgs mediated processes. The QGC has been reported for the first time by ATLAS collaboration using the VBS process, yielding a final state with two same sign $\mathrm{W}$ boson in association with two jets [4] in a purely electroweak process and also measured the $W \gamma \gamma$ production cross section [5] which is now accessible with the $8 \mathrm{TeV}$ LHC data set. The CMS collaboration has also measured the QGC in the $W W \gamma$ and $W Z \gamma$ final states [6]. So far the observations are consistent with SM predictions and as the sensitivity of these measurements improves, the TGC and QGC that lead to tri-boson final states can not only test the electroweak sector of the SM but also probe new physics. In this paper we look at some of the tri-boson production processes merged to 1 -jet in the warped extra dimension model.

In the model proposed by Randall and Sundrum (RS) [7], the non-factorizable geometry of the space-time with the inclusion of a single warped extra spatial dimension, proposes a solution to the hierarchy problem. The SM fields are confined to a 3-brane, whereas the gravity which propagates the full 5-dimensional space-time, manifests as massive Kaluza-Klein (KK) modes in 4-dimensional space-time. RS model phenomenology of the virtual graviton exchange have extensively been studied for gauge boson pair production processes viz., $\gamma \gamma$ [8], $Z Z$ [9], $W^{+} W^{-}$[10] and also for DY production [11] at the next-to-leading order (NLO) accuracy, because 
of their rich sensitivity to the model parameters. This in turn helps to reduce the theoretical uncertainties whereby constraining the RS model parameters. Recently the parton shower effect has been considered for those processes in [12]. Nevertheless, the study of triple gauge boson production processes within this model would also be phenomenologically important, as they could effectively participate in interesting new physics searches at the TeV scale. They have been studied in the SM at NLO [13, 14, 15, 16] level. NLO results of the triple photon production in the SM have recently been presented including the effect of photon fragmentation [17] or matching them with different parton shower (PS) Monte Carlo programmes [18]. These SM processes also serve as potential backgrounds to a number of new physics signals coming from different BSM scenarios. For example, the SM $\gamma \gamma \gamma$ process is a background to single photon production, together with one techni-pion in technicolor model, whereas $\gamma \gamma Z$ process in the SM is a background to the signal with di-photon plus missing energy in gauge-mediated supersymmetric theories.

In this analysis, we consider the production of neutral triple electroweak gauge bosons in warped extra dimension model at the LHC, i.e., $P P \rightarrow V V V X$, where $V=\gamma, Z$ and $X$ denotes some hadronic final states. Similar processes have been analysed at LO in case of large extra dimensional models [19] [20] [21]. In fact, study of these processes in RS scenario bears equal importance, as their contributions in searching new physics using the triple gauge boson productions are undeniable in distinguishing physics arising from the potential BSM candidates like supersummetry or technicolor.

This paper is organised in the following way: in section 2 we present brief description of the RS model. In section 3 we discuss the merging procedure and computational details. Numerical results of various kinematical distributions are provided in section 4 and finally we draw the conclusion of our study in the last section.

\section{Neutral Triple Vector Boson Production in RS Model}

In the RS model, the non-factorizable geometry is governed by the following 5dimensional warped metric [22],

$$
d s^{2}=e^{-2 \kappa r_{c}|\phi|} \eta_{\mu \nu} d x^{\mu} d x^{\nu}-r_{c}^{2} d \phi^{2} \quad,
$$

where $\eta_{\mu \nu}$ represents the flat Minkowski metric. $\phi$ denotes the fifth dimension $(0 \leq$ $\phi \leq \pi$ ) which is compactified on a $S^{1} / \mathbb{Z}_{2}$ orbifold with a radius $r_{c}$ and $\kappa$ is related to the curvature of the $A d S_{5}$ space-time. Two 3-branes with opposite tensions are situated on two fixed points $(\phi=0, \pi)$ of the extra dimension. The brane at $\phi=0$ is called the 'Planck brane' and the other one at $\phi=\pi$ is known as the 'TeV brane'. In the RS scenario, all SM fields are considered to be confined on the $\mathrm{TeV}$ brane, whereas the gravity can propagate in the full $4+1$ dimensions. The interaction 
among the SM fields with the massive KK excitations $\left(h_{\mu \nu}^{(n)}\right)$ of the graviton [23, 24] is determined by the following Lagrangian,

$$
\mathcal{L}_{\mathcal{R S}}=-\frac{1}{\bar{M}_{P l}} T^{\mu \nu}(x) h_{\mu \nu}^{(0)}(x)-\frac{\bar{c}_{0}}{m_{0}} T^{\mu \nu}(x) \sum_{n=1}^{\infty} h_{\mu \nu}^{(n)}(x)
$$

where $\bar{c}_{0}=\frac{\kappa}{\bar{M}_{P l}}, m_{0}=\kappa e^{-\kappa r_{c} \pi}, T^{\mu \nu}$ is the energy-momentum tensor for the SM particles and $\bar{M}_{P l}$ is the reduced Planck scale. Note that, the couplings of the zeroth KK mode to the SM fields are $\bar{M}_{P l}$ suppressed and hence this term can be practically neglected. However, the contribution from the higher modes with the coupling $\bar{c}_{0} / m_{0}$ can be of the order of few $\mathrm{TeV}$ for a choice of $\kappa r_{c} \sim \mathcal{O}(10)$ [25, 26] and they can produce significant observable effects. The masses of the KK mode excitations are given by $M_{n}=x_{n} \kappa e^{-\pi \kappa r_{c}}$, where $x_{n}$ indicates to the zeros of the Bessel function $J_{1}(x)$.

The effective graviton propagator after summing over all the massive KK modes except the zeroth one takes the following form [27] [8],

$$
\begin{aligned}
D_{e f f}\left(s_{i j}\right) & =\sum_{n=1}^{\infty} \frac{1}{s_{i j}-M_{n}^{2}+i \Gamma_{n} M_{n}} \\
& =\frac{1}{m_{0}^{2}} \sum_{n=1}^{\infty} \frac{\left(x^{2}-x_{n}^{2}\right)-i x_{n} \frac{\Gamma_{n}}{m_{0}}}{\left(x^{2}-x_{n}^{2}\right)^{2}+x_{n}^{2}\left(\frac{\Gamma_{n}}{m_{0}}\right)^{2}}
\end{aligned}
$$

where $s_{i j}=\left(p_{i}+p_{j}\right)^{2}, x=\sqrt{s_{i j}} / m_{0}$ and $\Gamma_{n}$ denotes the width of the resonance with mass $M_{n}$. The total decay width of the graviton can be calculated with the KK states decaying to the SM particles [24] 28] in the following way,

$$
\Gamma_{n}=m_{0} \bar{c}_{0}^{2} x_{n}^{3} \Delta_{n} \quad,
$$

where $\Delta_{n}$ is given by,

$$
\Delta_{n}=\Delta_{n}^{\gamma \gamma}+\Delta_{n}^{Z Z}+\Delta_{n}^{W W}+\Delta_{n}^{H H}+\sum_{\nu} \Delta_{n}^{\nu \nu}+\sum_{l} \Delta_{n}^{l l}+\Delta_{n}^{g g}+\sum_{q} \Delta_{n}^{q q}
$$

Here each $\Delta_{n}^{a a}$ corresponds to the coefficient coming from the decay width calculation of the process $h^{(n)} \rightarrow a a$. Unlike the large extra dimension model, the individual resonances of the graviton are well-separated in the RS model and they can be probed in invariant mass distribution.

The massive RS graviton could be produced in association with a photon or a $Z$ boson and since the RS gravitons also couple to two photons or two $Z$ bosons, the SM three neutral gauge boson final state distributions could be altered due to the RS contributions and its interference with the SM. 


\section{Merging Matrix Element with Parton Shower}

The leading order neutral triple gauge boson production processes $P P \rightarrow V V V X$ at the LHC come from the subprocess,

$$
q\left(p_{1}\right)+\bar{q}\left(p_{2}\right) \rightarrow V\left(p_{3}\right)+V\left(p_{4}\right)+V\left(p_{5}\right) \quad,
$$

where $V=\gamma, Z$ and $X$ is any final state hadron. This process has been merged with the 1-jet process $P P \rightarrow V V V j X$ in MadGraPh5 (MG) 29] framework to have a better description of different distributions. Due to extra radiation emission, $q(\bar{q}) g$ initiated subprocesses also come up. The merged events are then matched to a Parton Shower (PS). The $Z$ bosons are let to decay to lepton pairs, thus accounting for the off-shell contributions.

In LHC, additional jets are often produced from initial state radiation and can alter the LO predictions for relevant observables. Generally these additional jets are simulated using PS monte carlo. But these QCD radiations in the PS programs are generated in the soft and collinear approximation based on Sudakov form factors. The widely separated and hard emissions are not well-described in the PS approach, whereas the fixed order tree level amplitudes can provide reliable predictions in the hard region, but it fails in the collinear and soft limits. Therefore it is also essential to take into account the tree level amplitude containing additional jets. Both descriptions have to be combined in an appropriate matching method by avoiding double counting or gaps between samples with different multiplicity. Several algorithms have been proposed for this purpose, mainly based on the event re-weighting (eg. CKKW) [30] [31] or event rejection (eg. MLM) [32].

The shower- $\mathrm{k}_{T}$ scheme [33], based on event rejection like MLM as implemented in MADGRAPH5 is used in this analysis. In this scheme the events are generated by MG with a minimum separation in the phase space $Q_{c u t}$ and $P_{T_{\min }}$ between the final-state partons $(i j)$ and between the final-state and initial-state partons $(i B)$ respectively which is characterized by the $k_{T}$ jet measure:

$$
d_{i j}^{2}=\min \left(p_{T_{i}}^{2}, p_{T_{j}}^{2}\right) \Delta R_{i j}^{2}>Q_{c u t}^{2}, \quad d_{i B}^{2}=p_{T_{i}}^{2}>p_{T_{m i n}}^{2}
$$

Here $\Delta R_{i j}^{2}=2\left[\cosh \left(\eta_{i}-\eta_{j}\right)-\cos \left(\phi_{i}-\phi_{j}\right)\right]$, where $p_{T_{i}}, \eta_{i}, \phi_{i}$ are the transverse momentum, pseudo-rapidity and azimuthal angle of the parton $i$. The $k_{T}$ value is set as the renormalization scale at each QCD emission vertex. The events are then passed to PYTHIA 34] for showering. In shower- $k_{T}$ scheme, pythia $p_{T}$-ordered shower is used for showering. PYTHIA reports the scale of the hardest emission $\left(Q_{\text {hardest }}^{P S}\right)$ in the shower and vetoes events based on the $k_{T}$ values of the hardest shower emission instead of performing a jet clustering and comparing to the ME. If $Q_{\text {hardest }}^{P S}>Q_{\text {cut }}$ for lower multiplicity samples, then the event is rejected, whereas for highest multiplicity sample an event is rejected if $Q_{\text {hardest }}^{P S}>Q_{\text {softest }}^{M E}$, the scale of the softest parton in the event from ME. We choose to work with $Q_{c u t}=p_{T_{\min }}$.

The fixed order merging approach gives a better description of the region of hard and well separated jet whereas the parton shower takes care of the infrared 
region correctly. These merged-matched events provide a realistic framework to be compared to the experimental outcomes. The Lagrangian of the RS model is written using FeynRules [35] and it is combined together with the SM Lagrangian. The universal FeynRules output (UFO) of the combined Lagrangian $\left(\right.$ i.e., $\mathcal{L}_{\mathcal{R S}}+\mathcal{L}_{\mathcal{S M}}$ ) is then imported within MADGRAPH5 framework and used for the generation of events. The model parameters $\bar{c}_{0}$ and $M_{1}$, the mass of the first excited KK mode have been set as external inputs and we choose to work with the following values: $M_{1}=1.7 \mathrm{TeV}$ and $\bar{c}_{0}=0.03$ which remain within the latest experimental bounds provided by ATLAS [36] 37] and CMS [38] 39] collaborations. In addition, we have systematically implemented the KK mode summation algorithm in the spin-2 HELAS routine [40]. Kinematical distributions of various observables have been recalculated for different di-final states such as, di-photon, Drell-Yan, $Z Z, W^{+} W^{-}$ in fixed order NLO and NLO+PS using this present layout and they are found to be in excellent agreement with those results that are present in the literature [8, 9, 10, 11, 12. This essentially ensures the proper execution of the whole computational set-up. We have generated events for the following four neutral triple vector boson production processes: (i) $\gamma \gamma \gamma$, (ii) $\gamma \gamma Z$, (iii) $\gamma Z Z$ and (iv) $Z Z Z$ under the above mentioned arrangements. The $Z$ bosons are decayed to lepton-pair which will be discussed in the next section in detail. Each of these processes consists of three types of contributions coming from pure SM, pure RS and the interference between these two.

\section{Numerical Result}

In this section, we present numerical results of various kinematical distributions for the above four processes. All the results are presented for LHC with center of mass energy $\sqrt{S}=13 \mathrm{TeV}$. In our analysis, the following set of external parameters are used as input:

$$
\begin{array}{ccc}
m_{Z}=91.188 \mathrm{GeV}, \quad \sin ^{2}\left(\theta_{W}\right) & =0.222, \\
G_{F}=1.16639 \cdot 10^{-5} \mathrm{GeV}^{-2}, & \alpha^{-1}=132.507 .
\end{array}
$$

During the generation of events we let the $Z$ bosons to decay to $\ell^{+} \ell^{-}$pair. Events are generated with loose cuts on the transverse momentum $\left(P_{T}\right)$ and rapidity $(y)$ of the final state particles:

$$
P_{T}^{\gamma, \ell}>15 \mathrm{GeV}, \quad\left|y^{\gamma, \ell}\right| \leq 2.6
$$

The factorization scale $\left(\mu_{F}\right)$ as well as the renormalization scale $\left(\mu_{R}\right)$ are chosen as the invariant mass of the final state particles and MSTW2008LO $(68 \% C L)$ PDF has been used. Throughout this paper, we have considered five massless quark flavors $\left(n_{f}=5\right)$ and neglected all top quark contributions. In case of processes containing two or three photons in final state, a photon separation cut $\Delta R_{\gamma \gamma}>0.3$ is used during event generation. $\Delta R_{\gamma \gamma}=\sqrt{(\Delta y)^{2}+(\Delta \phi)^{2}}$ is the separation of the 
two photons in the rapidity-azimuthal angle $(y, \phi)$ plane. For processes involving leptons and photons in final state, we have applied $\Delta R_{\gamma \ell}>0.3$ and $\Delta R_{\ell^{+} \ell^{-}}>0.3$. For consistently merging 0 -jet sample with 1 -jet sample we have followed the path prescribed in [33] and checked the stability of the cross-section by varying the scale $Q_{c u t}$. Additional checks on the smoothness of the distributions, for example the differential jet-rate (DJR) plots, in the ME and PS transition region have been done. For the four processes under consideration the choices of $Q_{c u t}$ are much the same around 90 - $95 \mathrm{GeV}$ where we find best smooth DJR plots and also the matched crosssection remains within $13 \%$ of the unmatched cross-section. For $\gamma \gamma \gamma, \gamma Z Z$ and $Z Z Z$ we choose $Q_{c u t}=95 \mathrm{GeV}$ whereas for $\gamma \gamma Z$ we took $Q_{c u t}=90 \mathrm{GeV}$. Showering is done with Pythia $P_{T^{-}}$ordered shower as described in the previous section. Different analysis cuts used on the final state particles at the time of analysis are described in the following subsections for each processes.

For the processes involving photons, the photons can come from the hard process or as a result of fragmentation which is a QED collinear effect. In order to get rid of such collinear divergences without involving additional non-perturbative effects, the smooth cone isolation criteria on the photons as proposed by Frixione [41], is used. A cone of radius $R=\sqrt{\left(y-y_{\gamma}\right)^{2}+\left(\phi-\phi_{\gamma}\right)^{2}}$ is considered in the $(y-\phi)$ plane around a photon satisfying the condition that the total hadronic transverse energy $E(R)$ within $R<R_{\gamma}$ would be less than a maximum limit $E(R)_{\max }$ given by,

$$
E(R)_{\max }=\epsilon_{\gamma} E_{T}^{\gamma}\left(\frac{1-\cos R}{1-\cos R_{\gamma}}\right)^{n} \quad,
$$

where $E_{T}^{\gamma}$ is the transverse energy of the photon; $\epsilon_{\gamma}, R_{\gamma}, n$ are three parameters of the Frixione isolation. During event generation we choose $\epsilon_{\gamma}=1, R_{\gamma}=0.3, n=1$.

For the reconstruction of $Z$ bosons from opposite sign lepton-pair, events are selected based on the selection criterion:

$$
\left|M_{\ell^{+} \ell^{-}}-M_{Z}\right| \leq 15 \quad G e V
$$

where $M_{\ell^{+} \ell^{-}}$is the reconstructed invariant mass of the opposite sign lepton-pair. The following transverse momentum and pseudo-rapidity cut on jets during analysis in all four processes are used

$$
P_{T}^{j}>50 \mathrm{GeV}, \quad \eta^{j} \leq 4.5
$$

For all four processes, an extra cut has been put on the final state particles invariant mass $(M>600 \mathrm{GeV})$ for transverse momentum distributions and rapidity distributions which are displayed in the respective figures. We have checked that the generated events give unbiased results with reference to the choices of generation and analysis cuts. Scale dependencies are obtained by varying the renormalization scale $\left(\mu_{R}\right)$ and the factorization scale $\left(\mu_{F}\right)$ [42] in the range $\left(\mu_{R}, \mu_{F}\right)=\left(\kappa_{R} \mu_{0}, \kappa_{F} \mu_{0}\right)$, where $\mu_{0}$ is the invariant mass of the three vector boson final states or its decay 
products when $Z \mathrm{~s}$ are involved. The scale factors $\kappa_{R}, \kappa_{F}$ are in the range $(1 / 2,2)$, we choose the combination $\left(\kappa_{R}, \kappa_{F}\right)=(1 / 2,1 / 2),(1,1),(1 / 2,1),(1,1 / 2),(1,2),(2,1)$, $(2,2)$ to study the scale variation. The scale uncertainty is represented by taking the envelope of all individual variations.
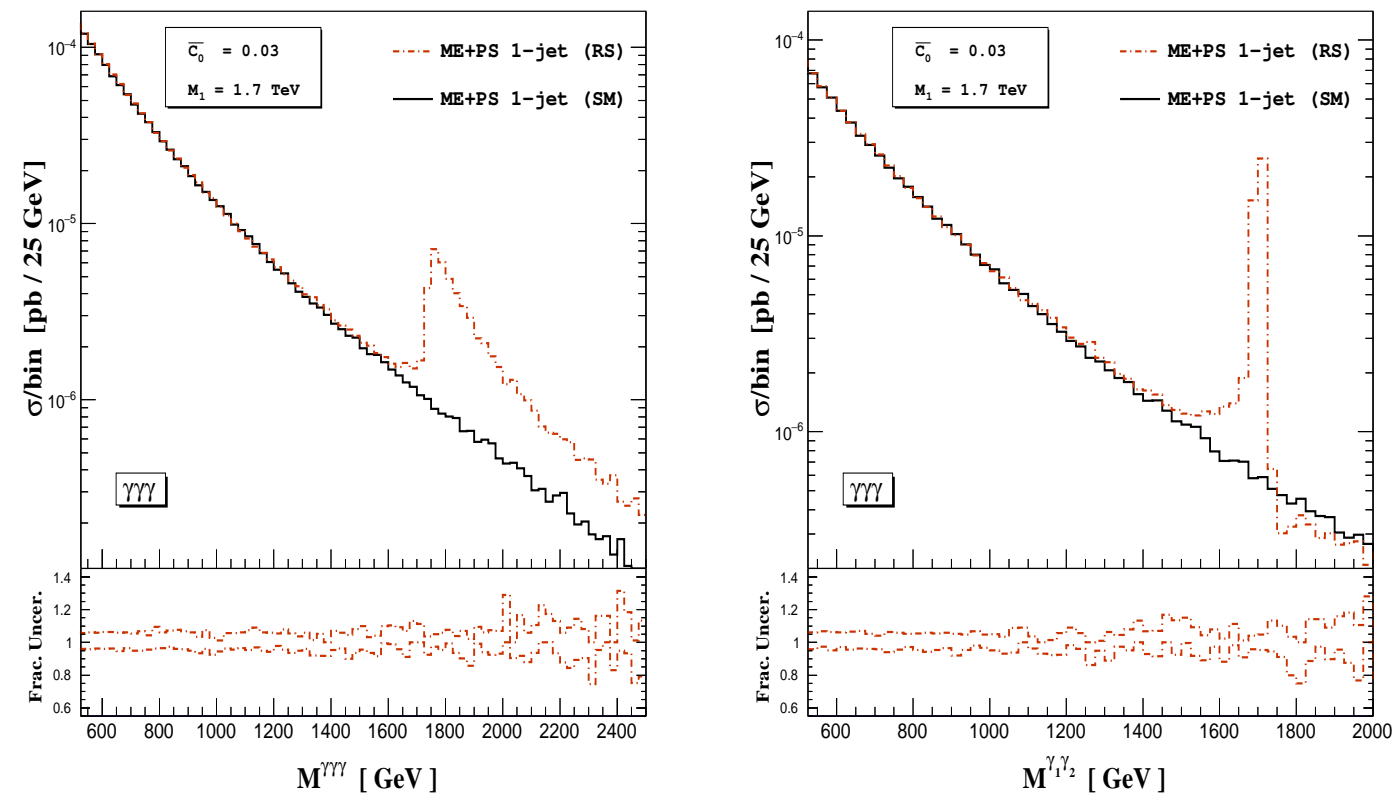

Figure 1: Invariant masses of $\gamma \gamma \gamma$ (left) and of two hardest photons (right) for $\gamma \gamma \gamma$ production.

Next we discuss and present our results for each of the neutral triple boson final states separately presenting some select distribution that are of interest for the RS model. For all figures, we followed the following convention; we give the distributions corresponding to the $\mathrm{SM}$ and $\mathrm{SM}+\mathrm{RS}$ that contribute to the observable for $\mathrm{ME}+\mathrm{PS}$ merged to 1 -jet for central scale choice $\left(\kappa_{R}, \kappa_{F}\right)=(1,1)$. In the lower inset we put the fractional scale uncertainty for ME+PS with 1-jet for RS case.

\section{$4.1 \gamma \gamma \gamma$}

Observing the $\gamma \gamma \gamma$ channel has a great advantage over the other triple neutral channels, because experimentally it provides a cleaner signature. $\gamma \gamma \gamma$ production in the RS model has also been studied in [43]. Here we present the result merged with 1-jet as well as include showering thus improving the result, viable to the experimental search. During the analysis level we use more stringent cuts than that used at the generation level: (a) cuts on transverse momentum and pseudo-rapidity of final state photons used are $P_{T}^{\gamma}>25 \mathrm{GeV}, \eta^{\gamma} \leq 2.5$, (b) Frixione parameters used are $R_{\gamma}=0.4, \epsilon_{\gamma}=1, n=2$ and (c) photon-photon separation cut $R_{\gamma \gamma}>0.4$. Finally the photons are ordered according to their transverse momentum. 

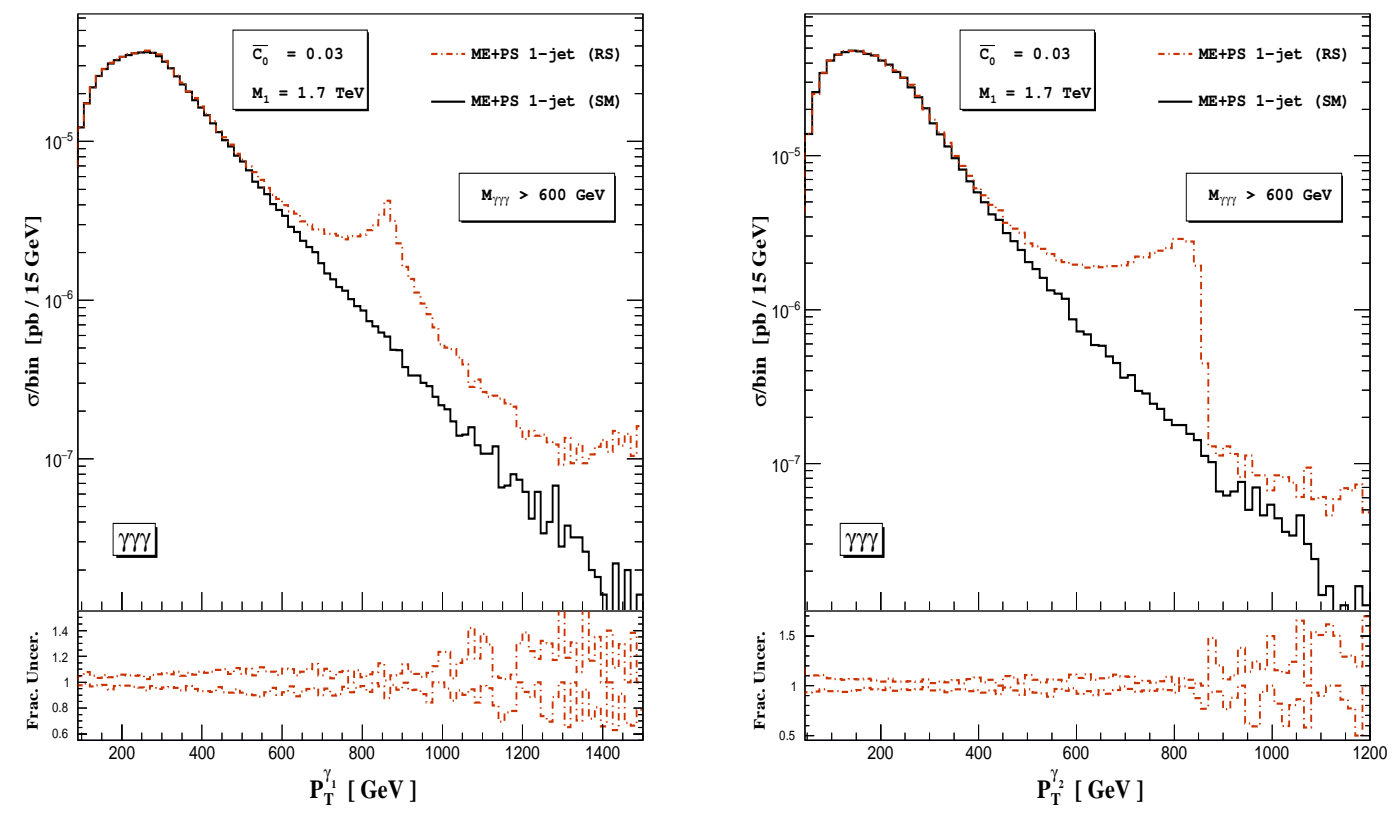

Figure 2: Transverse momentum distributions of hardest photon $\gamma_{1}$ (left) and next hard photon $\gamma_{2}$ (right) for $\gamma \gamma \gamma$ production.
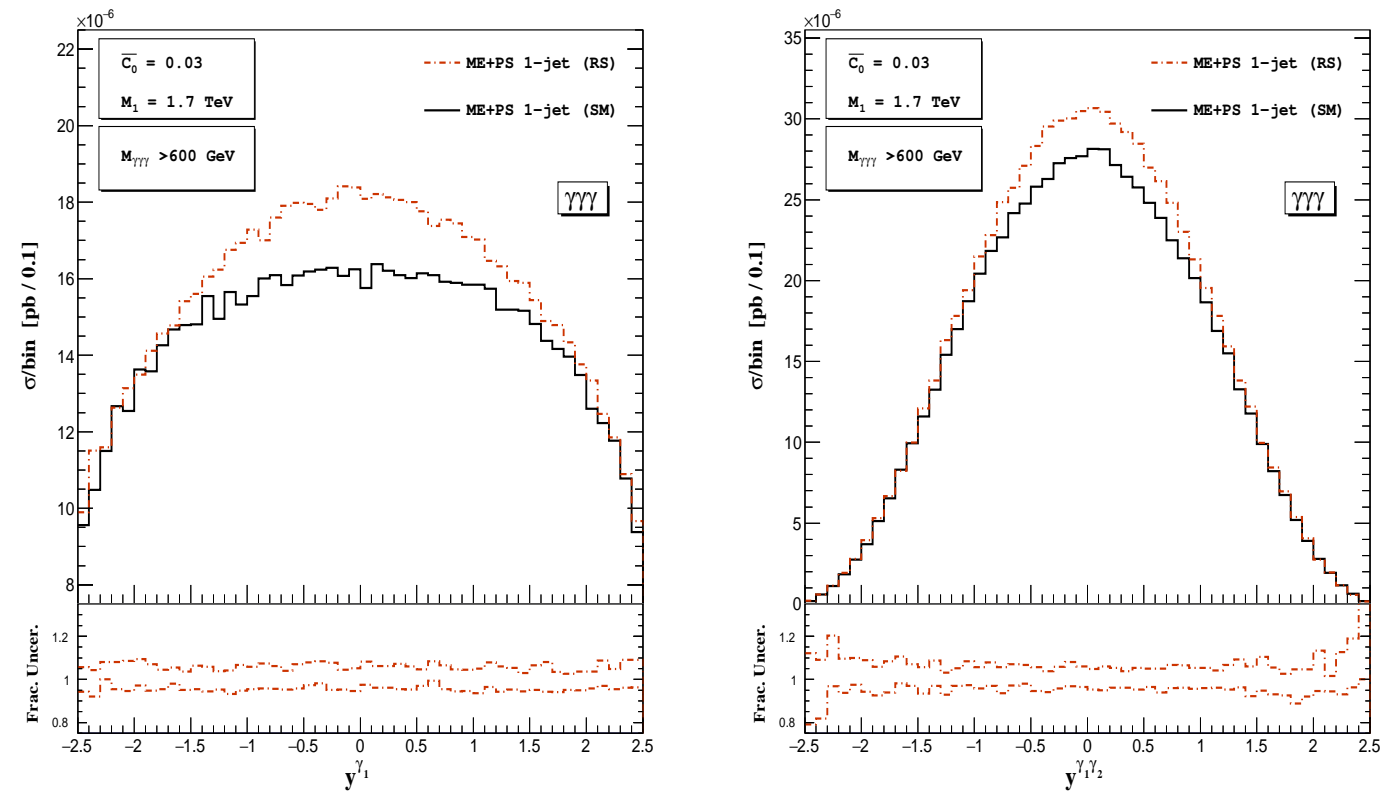

Figure 3: Rapidity distributions of hardest photon (left) and hardest pair (right) for $\gamma \gamma \gamma$ production.

In the Fig. 1, we present the invariant mass distribution for tri-photon on the left panel and the invariant mass of the hardest di-photon state on the right panel. In the invariant mass distribution of the tri-photon the peak appears near $1.7 \mathrm{TeV}$. 
The peak is slightly shifted from $1.7 \mathrm{TeV}$ towards higher invariant mass region. This is evident from the fact that the RS graviton is produced in association with a vector boson. In the expression for invariant mass there are dot products between all three momenta of the final state photons, thus shifting the peak slightly towards the higher invariant mass region. A cleaner signature of RS graviton can be found in the invariant mass distribution of the hardest two photon pairs where the RS peak appears at $1.7 \mathrm{TeV}$. From the transverse momentum distributions (Fig. 2) as well as in the rapidity distributions (Fig. 3), significant deviation from SM results is observed. In general the 1-jet merged sample gives a harder distributions. For the Fig. 1 (left panel) the uncertainty at the RS peak is about $7.7 \%$ and for the di-photon invariant mass (right panel) the uncertainty is about $10 \%$. For the $p_{T}$ distribution the uncertainties are larger, for the hardest photon (Fig. 21) it is about $15 \%$ and for the second hardest it is about $8.3 \%$ around the peaks in the $P_{T}$ distribution which correspond to about half the RS resonance. For the rapidity plots (Fig. 3) the uncertainty is about $10 \%$ in the central rapidity region.

From the invariant mass distributions (Fig. 1) an estimation of the signal and background events for the LHC Run-II can be made. For this purpose we have considered the invariant mass distributions over $1500 \mathrm{GeV}$ where the enhancement due to RS graviton signal is clearly visible over the SM background. We find that for $100 \mathrm{fb}^{-1}$ luminosity there are 8 signal events over 3 background events from the $M^{\gamma \gamma \gamma}$ distribution (Fig. 1 left panel) and 6 signal events over 2 background events from $M^{\gamma_{1} \gamma_{2}}$ distribution (Fig. 1 right panel).

\section{$4.2 \quad \gamma \gamma Z$}

In $\gamma \gamma Z$ production the effect of the massive RS KK-modes can be observed in the $\gamma \gamma Z$ invariant mass as well as in the di-photon invariant mass (Fig. 44). The $Z$ boson is allowed to decay to $\mu^{+} \mu^{-}$pair. Minimal analysis level cuts on the final state leptons and photons transverse momentum $P_{T}^{\gamma, l} \geq 25 \mathrm{GeV}$ and pseudo-rapidity cut $\eta^{\gamma, l} \leq 2.5$ are put. In order to fulfill detector resolution, cuts on the separation of photon, lepton and jets are imposed:

$$
R_{\gamma l}>0.4, \quad R_{l l}>0.4, \quad R_{l j}>0.7 .
$$

The invariant mass distribution of $\gamma \gamma \mu^{+} \mu^{-}$system shows an enhancement at about the RS peak but is additionally shifted compared to the tri-photon case due to the mass of the $Z$ boson. In this process the RS graviton can only decay into a photonpair, hence the invariant mass distribution of di-photon pair shows the peak at the RS mass. The uncertainty in the enhanced region is about $8 \%$ for the $\gamma \gamma \mu^{+} \mu^{-}$ invariant mass distribution and for the di-photon invariant mass at the RS peak it is about $9.9 \%$. The transverse momentum distributions (Fig. 5) of each photon clearly shows the RS signature appearing nearly at the half of the KK mass. The uncertainty for the transverse momentum distribution are much higher, at about $26 \%$. 

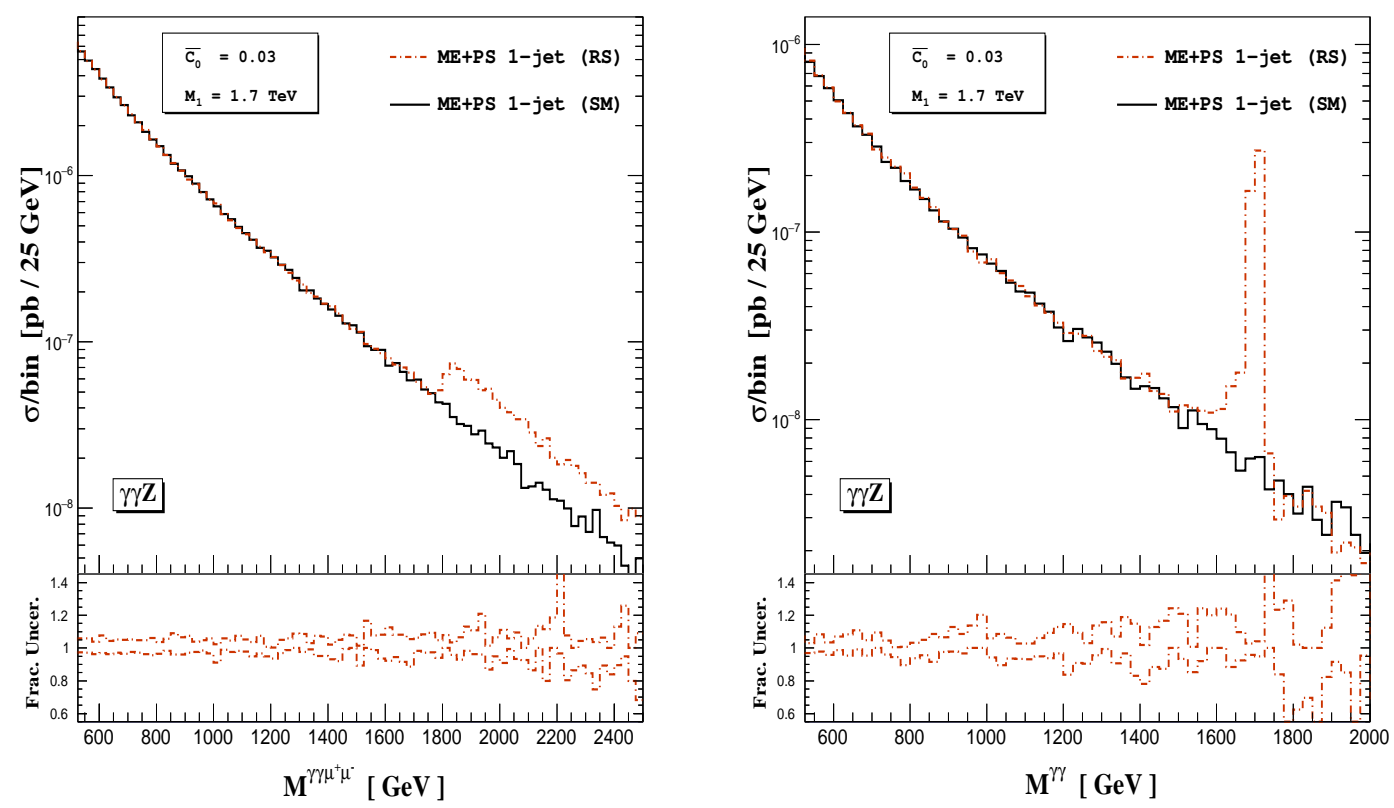

Figure 4: Invariant mass distributions of $\gamma \gamma \mu^{+} \mu^{-}$(left) and $\gamma \gamma$ (right) for $\gamma \gamma Z$ production.
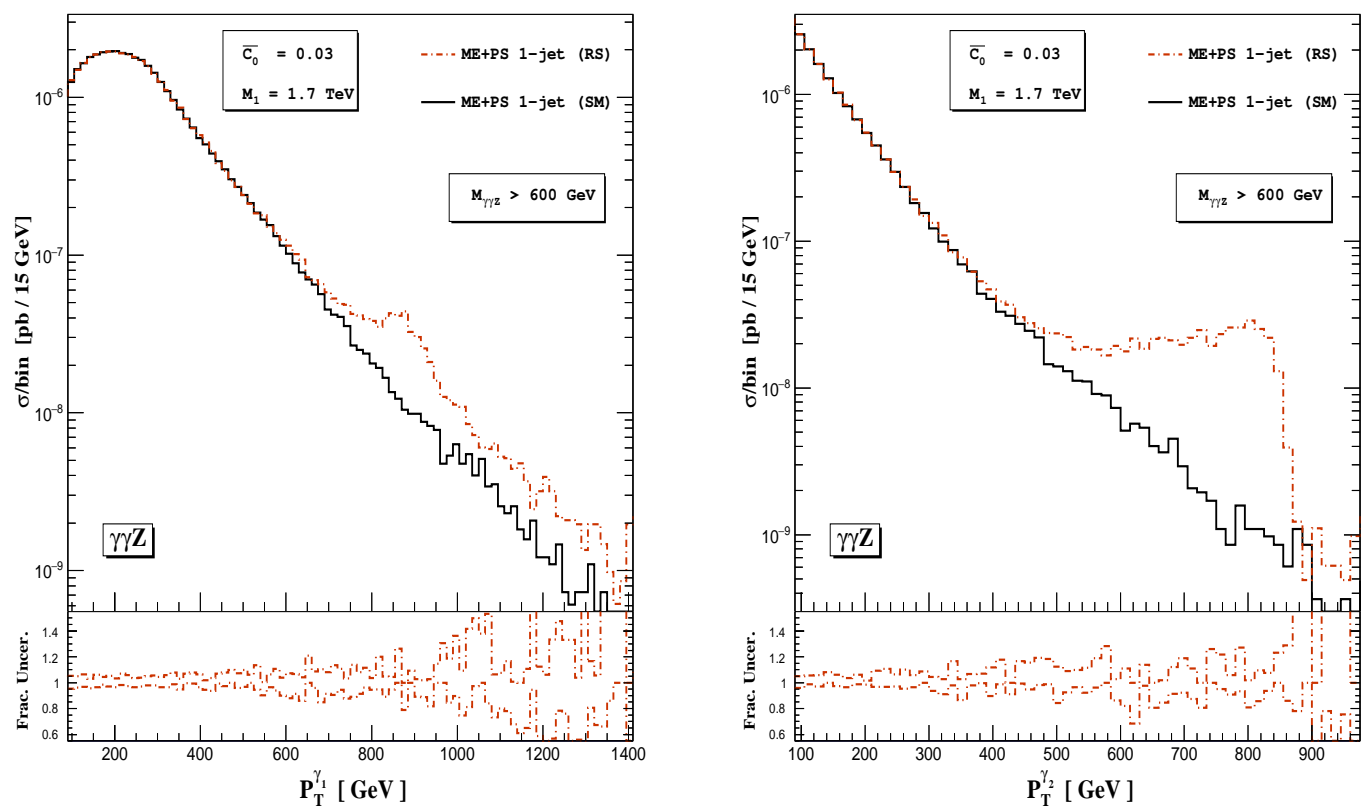

Figure 5: Transverse momentum distributions of hardest photon $\gamma_{1}$ (left) and second hard photon $\gamma_{1}$ (right) for $\gamma \gamma Z$ production.

In the $P_{T}$ distributions in Figs. 2, 5 the parton shower resums the large logarithms in the collinear region which suppress the low $P_{T}$ cross section. In this region the scale uncertainty is also low as the higher logarithms are resummed to all orders. 

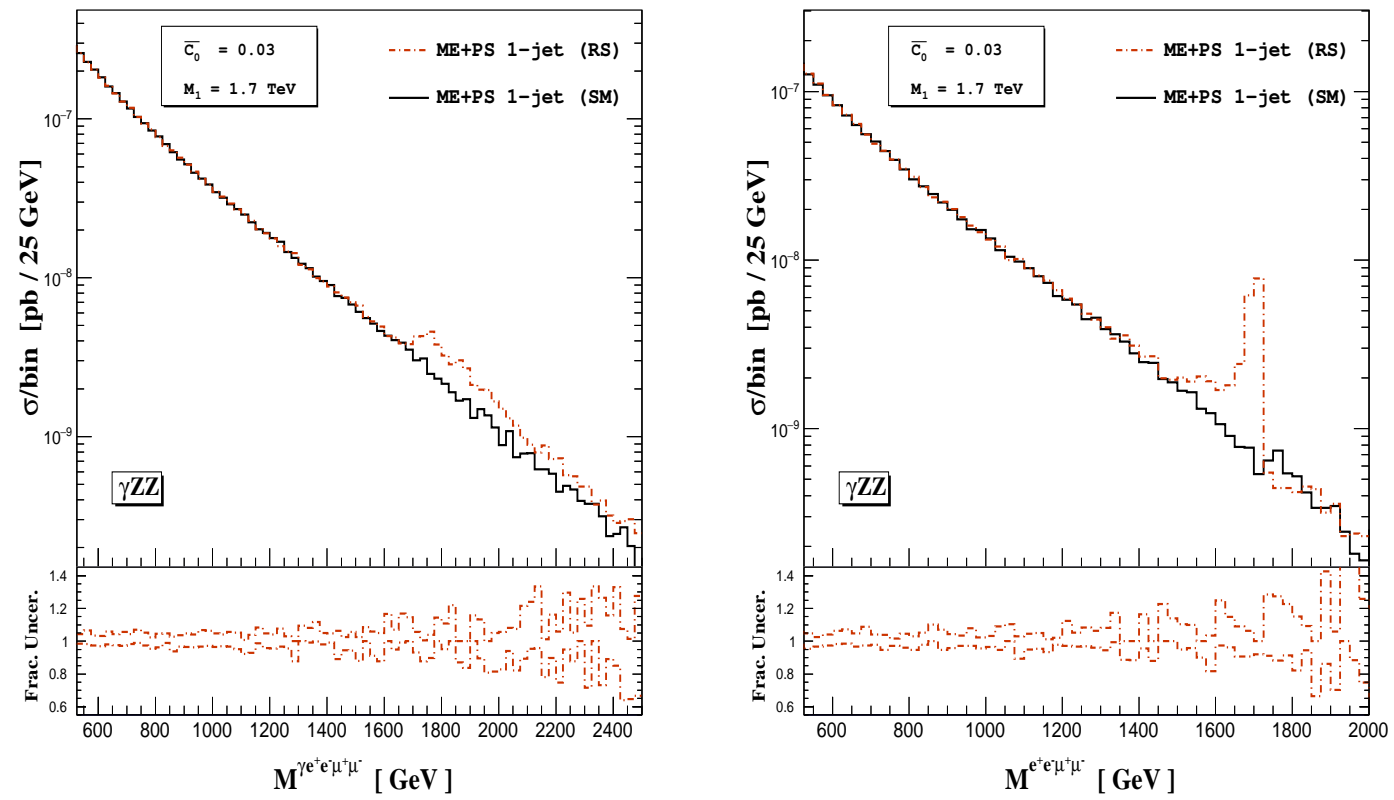

Figure 6: Invariant mass distributions of $\gamma e^{+} e^{-} \mu^{+} \mu^{-}$(left) and $e^{+} e^{-} \mu^{+} \mu^{-}$(right) for $\gamma Z Z$ production.

The same set of analysis cuts are used here, as the $\gamma \gamma Z$ case. One $Z$-boson is decayed to a $e^{+} e^{-}$pair and the other $Z$-boson to a $\mu^{+} \mu^{-}$pair during event generation. In the $\gamma Z Z$ invariant mass distribution (Fig. 6) there is slight enhancement of $\mathrm{RS}$ contribution over the SM, around the invariant mass region $(1.7 \mathrm{TeV})$. But the effect of massive KK state is best found on the di- $Z$ boson invariant mass distribution (Fig. 6) and it peaks at $1.7 \mathrm{TeV}$ as expected. The $p_{T}$ distributions are not very useful to discriminating the RS signatures. The uncertainty for the invariant mass distribution of $\gamma e^{+} e^{-} \mu^{+} \mu^{-}$system is about $15 \%$ and for the invariant mass of the $e^{+} e^{-} \mu^{+} \mu^{-}$system which is as a result of the RS graviton decay is about $8.8 \%$.

\section{$4.4 \quad Z Z Z$}

For $Z Z Z$ production, we choose to decay two $Z$ bosons to two $\mu^{+} \mu^{-}$pairs whereas the other $Z$ boson is decayed to a $e^{+} e^{-}$pair. For this process also same set of analysis cuts has been used, $P_{T}^{l} \geq 25 \mathrm{GeV}, \eta^{l} \leq 2.5$, where $l=e^{+}, e^{-}, \mu^{+}, \mu^{-}$. The $Z$ bosons are reconstructed according to the criterion described at the beginning of this section (see Eq. (10) ). The reconstructed $Z$ bosons are then ordered according to their transverse momentum. Thus in the figures $Z_{1,2}$ represents transverse momentum ordered $Z$ bosons. Small enhancement over the SM can be seen in the $Z Z Z$ invariant mass distribution (Fig. 7 left panel). The uncertainty in that region is found to be about $13-14 \%$. In this case also the RS contribution is best found in the hardest 

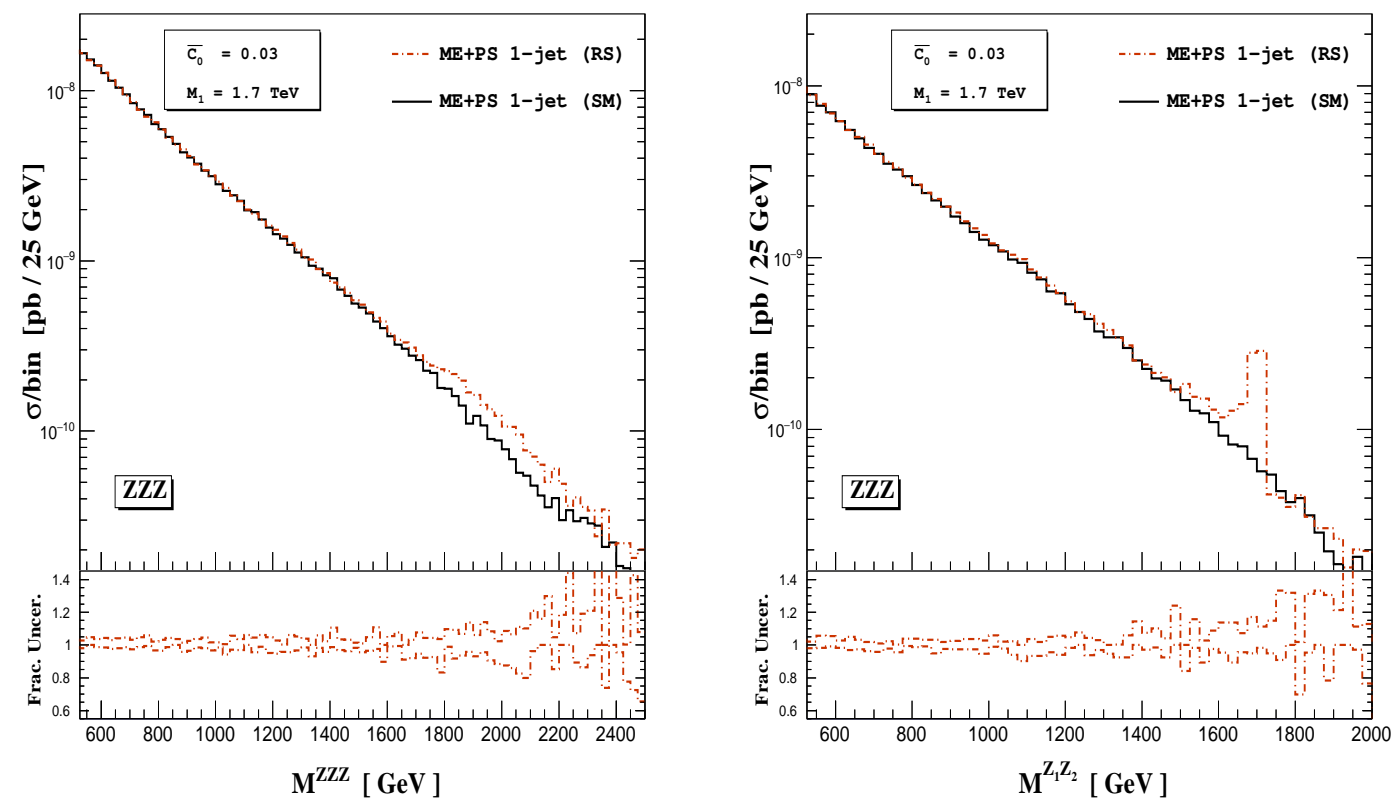

Figure 7: Invariant mass distributions of $e^{+} e^{-} \mu^{+} \mu^{-} \mu^{+} \mu^{-}$(left) and hardest two lepton pairs (right) for $Z Z Z$ production.

two $Z$ boson invariant mass distribution (Fig. 7 right panel). The uncertainty at the peak region is about $13.25 \%$.

\section{Conclusion}

In the context of RS model, effects of the exchange of virtual KK graviton have been studied on the neutral triple gauge boson production processes at the $13 \mathrm{TeV}$ LHC. This process could play a vital role in discriminating physics beyond the SM and in estimating the contribution coming from potential BSM scenarios in new physics searches. We have incorporated the RS model, using FeynRules in association with an algorithm that takes care of the KK mode summation of gravitons within MADGRAPH5 environment and performed a number of checks to ensure their proper implementation.

We have merged $P P \rightarrow V V V$ and $P P \rightarrow V V V+j$ event samples for better prediction of the distributions and observe that it gives harder distributions compared to the unmerged sample. To make theoretical prediction closer to the experimental situation, we have also matched the merged events with parton shower. Final state $Z$ bosons are allowed to decay to either of the following leptonic decay modes: $(i)$ $Z \rightarrow e^{+} e^{-}$, (ii) $Z \rightarrow \mu^{+} \mu^{-}$at the time of event generation, thus taking into account off-shell effects as well. For process with more than one stable photon, the photons are ordered according to their $P_{T}$ and then the required number of photons are collected, based on their hardness. Likewise, for triple $Z$-boson production, $Z$ bosons 
are reconstructed from their daughter particles and then ordered according to the hardness. Numerical results of some selective differential distributions for a set of kinematical variables have been presented for the merged samples. All these codes are flexible enough to incorporate the experimental cuts, different values for model parameters etc. and they can be used to obtain numerical results of any kind of distributions that would indeed help the experimental collaborations.

Of the neutral tri-gauge boson final states considered here, the tri-photon final state has the highest rate and can be used to look for signatures of the RS model. For the tri-photon process, the invariant mass, $P_{T}$ distributions of various photons ordered in terms of the hardness and rapidity distributions are all good discriminators of the RS model. The scale uncertainties are by and large within $10 \%$ for the invariant mass distribution. In the tri-final invariant mass distribution the cross section is enhanced in the $\mathrm{RS}$ resonance peak region, which diminishes in going from the $\gamma \gamma \gamma$ to the $Z Z Z$ final state. The di-invariant mass distribution for all four processes is a clear indicator of the RS resonance peak. In the di-invariant mass distributions the peak of the RS resonance is most enhanced for the $\gamma \gamma \gamma$ and diminishes in going to the $Z Z Z$ production process.

\section{Acknowledgements}

We acknowledge the high performance cluster computing facility of the Theory Division, SINP. The authors also thank Satyajit Seth for collaboration during the initial stages of this work. GD would like to thank Olivier Mattelaer, Paolo Torrielli for discussion regarding MADGRAPH5. GD also thanks Suvankar Roy Chowdhury, Atanu Modak of CMS for useful discussions. The authors would like to thank V. Ravindran for useful comments. Work of GD is supported by funding from Department of Atomic Energy, India.

\section{References}

[1] ATLAS Collaboration, G. Aad et al., Observation of a new particle in the search for the Standard Model Higgs boson with the ATLAS detector at the LHC, Phys. Lett. B716 (2012) 1-29, [arXiv:1207.7214].

[2] CMS Collaboration, S. Chatrchyan et al., Observation of a new boson at a mass of $125 \mathrm{GeV}$ with the CMS experiment at the LHC, Phys. Lett. B716 (2012) 30-61, arXiv:1207.7235.

[3] M. E. Peskin, On the Trail of the Higgs Boson, arXiv:1506.0818.

[4] ATLAS Collaboration, G. Aad et al., Evidence for Electroweak Production of $W^{ \pm} W^{ \pm} j j$ in pp Collisions at $\sqrt{s}=8$ TeV with the ATLAS Detector, Phys. Rev. Lett. 113 (2014), no. 14 141803, [arXiv:1405.6241]. 
[5] ATLAS Collaboration, G. Aad et al., Evidence of $W$ Production in $p p$ Collisions at $s=8 \mathrm{Te} V$ and Limits on Anomalous Quartic Gauge Couplings with the ATLAS Detector, Phys. Rev. Lett. 115 (2015), no. 3 031802, [arXiv:1503.0324].

[6] CMS Collaboration, S. Chatrchyan et al., Search for $W W \gamma$ and $W Z \gamma$ production and constraints on anomalous quartic gauge couplings in pp collisions at $\sqrt{s}=8$ TeV, Phys. Rev. D90 (2014), no. 3 032008, [arXiv:1404.4619].

[7] L. Randall and R. Sundrum, A Large mass hierarchy from a small extra dimension, Phys.Rev.Lett. 83 (1999) 3370-3373, hep-ph/9905221.

[8] M. Kumar, P. Mathews, V. Ravindran, and A. Tripathi, Direct photon pair production at the LHC to order $\alpha_{s}$ in TeV scale gravity models, Nucl.Phys. B818 (2009) 28-51, [arXiv:0902.4894].

[9] N. Agarwal, V. Ravindran, V. K. Tiwari, and A. Tripathi, Next-to-leading order QCD corrections to the $Z$ boson pair production at the LHC in Randall Sundrum model, Phys.Lett. B686 (2010) 244-248, [arXiv:0910.1551].

[10] N. Agarwal, V. Ravindran, V. K. Tiwari, and A. Tripathi, Next-to-leading order QCD corrections to $W^{+} W^{-}$production at the LHC in Randall Sundrum model, Phys.Lett. B690 (2010) 390-395, [arXiv:1003.5445].

[11] P. Mathews, V. Ravindran, and K. Sridhar, NLO-QCD corrections to dilepton production in the Randall-Sundrum model, JHEP 10 (2005) 031, hep-ph/0506158.

[12] G. Das, P. Mathews, V. Ravindran, and S. Seth, RS resonance in di-final state production at the LHC to NLO+PS accuracy, JHEP 1410 (2014) 188, [arXiv:1408.3970].

[13] G. Bozzi, F. Campanario, M. Rauch, and D. Zeppenfeld, $Z \gamma \gamma$ production with leptonic decays and triple photon production at next-to-leading order QCD, Phys.Rev. D84 (2011) 074028, [arXiv:1107.3149].

[14] T. Binoth, G. Ossola, C. Papadopoulos, and R. Pittau, NLO QCD corrections to tri-boson production, JHEP 0806 (2008) 082, [arXiv:0804.0350].

[15] G. Bozzi, F. Campanario, V. Hankele, and D. Zeppenfeld, NLO QCD corrections to $W+W$-gamma and $Z Z$ gamma production with leptonic decays, Phys.Rev. D81 (2010) 094030, [arXiv:0911.0438].

[16] A. Lazopoulos, K. Melnikov, and F. Petriello, QCD corrections to tri-boson production, Phys.Rev. D76 (2007) 014001, hep-ph/0703273. 
[17] J. M. Campbell and C. Williams, Triphoton production at hadron colliders, arXiv:1403.2641.

[18] M. Mandal, P. Mathews, V. Ravindran, and S. Seth, Three photon production to $N L O+P S$ accuracy at the $L H C$, arXiv:1403.2917.

[19] M. Kumar, P. Mathews, V. Ravindran, and S. Seth, Neutral triple electroweak gauge boson production in the large extra-dimension model at the $\mathrm{LHC}$, Phys.Rev. D85 (2012) 094507, [arXiv:1111.7063].

[20] R.-C. Jiang, X.-Z. Li, W.-G. Ma, L. Guo, and R.-Y. Zhang, Triple $Z^{0}$-boson production in large extra dimensions model at ILC, Chin.Phys.Lett. 29 (2012) 111101, [arXiv:1211.6800].

[21] H. Sun and Y.-J. Zhou, Neutral Triple Gauge Boson production in the large extra dimensions model at linear colliders, Phys.Rev. D86 (2012) 075003, arXiv:1209.2214].

[22] H. Davoudiasl, J. Hewett, and T. Rizzo, Phenomenology of the Randall-Sundrum Gauge Hierarchy Model, Phys.Rev.Lett. 84 (2000) 2080, hep-ph/9909255].

[23] G. F. Giudice, R. Rattazzi, and J. D. Wells, Quantum gravity and extra dimensions at high-energy colliders, Nucl.Phys. B544 (1999) 3-38, hep-ph/9811291].

[24] T. Han, J. D. Lykken, and R.-J. Zhang, On Kaluza-Klein states from large extra dimensions, Phys.Rev. D59 (1999) 105006, hep-ph/9811350.

[25] W. D. Goldberger and M. B. Wise, Modulus stabilization with bulk fields, Phys.Rev.Lett. 83 (1999) 4922-4925, hep-ph/9907447.

[26] W. D. Goldberger and M. B. Wise, Phenomenology of a stabilized modulus, Phys.Lett. B475 (2000) 275-279, hep-ph/9911457.

[27] H. Davoudiasl, J. Hewett, and T. Rizzo, Experimental probes of localized gravity: On and off the wall, Phys.Rev. D63 (2001) 075004, hep-ph/0006041.

[28] S. Kumar Rai and S. Raychaudhuri, Single photon signals for warped quantum gravity at a linear e+ e-collider, JHEP 0310 (2003) 020, hep-ph/0307096.

[29] J. Alwall, R. Frederix, S. Frixione, V. Hirschi, F. Maltoni, O. Mattelaer, H. S. Shao, T. Stelzer, P. Torrielli, and M. Zaro, The automated computation of tree-level and next-to-leading order differential cross sections, and their matching to parton shower simulations, JHEP 07 (2014) 079, arXiv:1405.0301]. 
[30] S. Catani, F. Krauss, R. Kuhn, and B. Webber, QCD matrix elements + parton showers, JHEP 0111 (2001) 063, hep-ph/0109231.

[31] F. Krauss, Matrix elements and parton showers in hadronic interactions, JHEP 0208 (2002) 015, hep-ph/0205283.

[32] J. Alwall, S. Hoche, F. Krauss, N. Lavesson, L. Lonnblad, et al., Comparative study of various algorithms for the merging of parton showers and matrix elements in hadronic collisions, Eur.Phys.J. C53 (2008) 473-500, arXiv:0706.2569.

[33] J. Alwall, S. de Visscher, and F. Maltoni, QCD radiation in the production of heavy colored particles at the LHC, JHEP 0902 (2009) 017, [arXiv:0810.5350].

[34] T. Sjostrand, S. Mrenna, and P. Z. Skands, PYTHIA 6.4 Physics and Manual, JHEP 05 (2006) 026, hep-ph/0603175.

[35] A. Alloul, N. D. Christensen, C. Degrande, C. Duhr, and B. Fuks, FeynRules 2.0 - A complete toolbox for tree-level phenomenology, arXiv:1310.1921.

[36] ATLAS Collaboration Collaboration, G. Aad et al., Search for Extra Dimensions in diphoton events using proton-proton collisions recorded at $\sqrt{s}=7$ TeV with the ATLAS detector at the LHC, New J.Phys. 15 (2013) 043007, [arXiv:1210.8389].

[37] ATLAS Collaboration Collaboration, G. Aad et al., Search for Extra Dimensions using diphoton events in $7 \mathrm{TeV}$ proton-proton collisions with the ATLAS detector, Phys.Lett. B710 (2012) 538-556, [arXiv:1112.2194].

[38] CMS Collaboration, V. Khachatryan et al., Search for resonances and quantum black holes using dijet mass spectra in proton-proton collisions at $\sqrt{s}=8$ TeV, Phys.Rev. D91 (2015), no. 5 052009, [arXiv:1501.0419].

[39] CMS Collaboration Collaboration, S. Chatrchyan et al., Search for signatures of extra dimensions in the diphoton mass spectrum at the Large Hadron Collider, Phys.Rev.Lett. 108 (2012) 111801, [arXiv:1112.0688].

[40] K. Hagiwara, J. Kanzaki, Q. Li, and K. Mawatari, HELAS and MadGraph/MadEvent with spin-2 particles, Eur.Phys.J. C56 (2008) 435-447, [arXiv:0805.2554].

[41] S. Frixione, Isolated photons in perturbative QCD, Phys.Lett. B429 (1998) 369-374, hep-ph/9801442.

[42] R. Frederix, S. Frixione, V. Hirschi, F. Maltoni, R. Pittau, and P. Torrielli, Four-lepton production at hadron colliders: aMC@NLO predictions with theoretical uncertainties, JHEP 02 (2012) 099, [arXiv:1110.4738]. 
[43] D. Atwood and S. K. Gupta, Probing Randall-Sundram Model using triphotons at the LHC, arXiv:1006.4370. 